\title{
The Effect of Nozzle Trailing Edge Thickness on Jet Noise
}

\author{
Brenda Henderson ${ }^{*}$, Kevin Kinzie ${ }^{\dagger}$, and Henry Haskin ${ }^{\ddagger}$ \\ NASA Langley Research Center Hampton, Virginia 23681
}

\begin{abstract}
The effect of nozzle trailing edge thickness on broadband acoustic radiation and the production of tones is investigated for coannular nozzles. Experiments were performed for a core nozzle trailing edge thickness between $0.38 \mathrm{~mm}$ and $3.17 \mathrm{~mm}$. The on-set of discrete tones was found to be predominantly affected by the velocity ratio, the ratio of the fan velocity to the core velocity, although some dependency on trailing edge thickness was also noted. For a core nozzle trailing edge thickness greater than or equal to $0.89 \mathrm{~mm}$, tones were produced for velocity ratios between 0.91 and 1.61. For a constant nozzle trailing edge thickness, the frequency varied almost linearly with the core velocity. The Strouhal number based on the core velocity changed with nozzle trailing edge thickness and varied between 0.16 and 0.2 for the core nozzles used in the experiments. Increases in broadband noise with increasing trailing edge thickness were observed for tone producing and non-tone producing conditions. A variable thickness trailing edge (crenellated) nozzle resulted in no tonal production and a reduction of the broadband trailing edge noise relative to that of the corresponding constant thickness trailing edge.
\end{abstract}

\section{Introduction}

The introduction of mixing devices to scale model nozzles can result in thickening of the nozzle trailing edges. Additionally, structural constraints in high temperature model testing can lead to thick nozzle trailing edges that do not scale to full scale with the other dimensions of the nozzle. Increases in high frequency broadband noise and the production of discrete frequency sound can occur when the core nozzle of a coannular nozzle system becomes excessively thick. The increased trailing edge noise in scale model tests can bias full-scale predictions made from these tests. The purpose of the present investigation is to determine the effect of core nozzle trailing edge thickness on the production of tones and the increase in high frequency broadband noise for externally plugged coannular nozzles.

Powell ${ }^{1}$ was perhaps one of the first to consider the effects of edge noise and used dimensional analysis to show that the acoustic power associated with edge noise scaled with the forth to the fifth power of the velocity. Ffowcs Williams and Hall ${ }^{2}$ showed analytically that, in a low Mach number flow, eddies in close proximity to the edge of a half plane radiate sound more effectively than those in free space. They showed that the acoustic intensity increased with the fifth power of the velocity. For airfoils, trailing edge noise ${ }^{3}$ results from interactions of turbulent or laminar boundary layers in close proximity to a thin trailing edge and from vortex shedding from a blunt trailing edge. The trailing edge noise scales with the fifth power of the velocity and the vortex shedding noise scales with the 5.5 power of the Mach number. For vortex shedding noise, the peak spectral levels increase with increasing trailing edge thickness.

Trailing edge noise associated with nozzle flow is not as well documented as that associated with flow over an airfoil possibly due to the fact that the acoustic radiation associated with jet noise scales on a much higher power of the jet velocity than that associated with trailing edge noise and, therefore, the jet noise is expected to dominate the acoustic radiation at high flow speeds. However, the effect of trailing edge noise in scale model tests can be significant ${ }^{4,5,6}$. Early analytical investigations ${ }^{7}$ into the acoustic field associated with turbulence near a jet exit assumed a negligible trailing edge thickness and showed that the acoustic intensity scaled with the sixth power of the velocity. Olsen et al. ${ }^{4}$ found narrowband high frequency "lip thickness" noise produced by coannular nozzles with velocity ratios above 0.5 , where the velocity ratio is defined as the ratio of the fan velocity to the core velocity. Discrete tones were not observed in these experiments. Olsen and $\mathrm{Karchmer}^{5}$ found significant narrowband lip

\footnotetext{
${ }^{*}$ Research Engineer, Mail Stop 166, AIAA Member

${ }^{\dagger}$ Senior Research Engineer, Mail Stop 166, Senior AIAA Member

* Engineer, Mail Stop 166
} 
noise from coannular nozzles with thick core nozzles that appeared to resemble aeolian tones and scaled with the sixth power of the velocity. The amplitude of the lip noise was affected by the velocity ratio. The blunt-edged plate experiments of Brinich et al. ${ }^{8}$ demonstrated differences in vortex shedding rates and vortex patterns behind the plate as the velocity ratio was decreased from unity which may explain the differences in acoustic radiation with velocity ratio noted by Olsen and Karchmer. Additional broadband increases may be expected when trailing edge tones are produced if the resulting Strouhal number and level of the discrete tone are within a range known to amplify jet mixing noise ${ }^{9}$.

The experiments reported here consider the acoustic radiation associated with a core nozzle trailing edge thickness between $0.38 \mathrm{~mm}$ and $3.17 \mathrm{~mm}$ for an externally plugged coannular nozzle. The on-set of tones as a function of velocity ratio and nozzle trailing edge thickness is documented. Measured increases in broadband noise for tone producing and non-tone producing conditions are reported for scale model data and the impact on data extrapolated to full-scale is documented. The effect of a variable thickness (crenellated) nozzle on broadband acoustic radiation and the production of tones is investigated.

\section{Experimental Approach}

The experiments were performed with the Jet Engine Simulator (JES) in the Low Speed Aeroacoustic Wind Tunnel (LSAWT) at NASA Langley Research Center. A schematic of the facility is shown in Figure 1. The LSAWT is an in-draft tunnel with a free jet to simulate forward flight effects. The inlet to the test section is an acoustically treated $1.43 \mathrm{~m}$ square nozzle. Fiberglass wedges are placed on the floor, ceiling, and walls of the test cell. The test section is $10.36 \mathrm{~m}$ long with cross sectional dimensions of $5.18 \mathrm{~m}$ by $5.18 \mathrm{~m}$ (measured from tip-to-tip of the wedges). The flow collector at the exit of the test section is acoustically treated.

A 28-element sideline microphone array, indicated in Figure 1, is located at $3.52 \mathrm{~m}$ from the centerline axis of the JES. Bruel and Kjaer type $49396.35 \mathrm{~mm}$ diameter condenser microphones are used for the acoustic measurements. The narrowband data contained in this paper are reported at the measurement location (microphone array) and have been corrected (to a standard day) for atmospheric absorption and shear layer effects ${ }^{10}$ unless otherwise specified.

The JES produces two independently controlled flow streams (core and fan) with maximum mass flow rates of $17 \mathrm{lbm} / \mathrm{s}$ in each stream. The simulator is equipped with two independently controlled propane sudden expansion burners to heat the core and fan streams separately. Each stream has electric pre-heat. The system is equipped with mufflers and flow straightening devices upstream of the nozzles. Total temperature and pressure rakes are located upstream of the nozzle contraction and are used to control nozzle pressure ratio (NPR), the ratio of the stagnation pressure to the pressure at the nozzle lip, and flow temperature. In the present investigation, only limited electric heat was used.

An externally plugged coannular nozzle system with a fan nozzle exit diameter of $23.9 \mathrm{~cm}$, a core nozzle exit diameter of $12.8 \mathrm{~cm}$, and a core nozzle exit area of $69.4 \mathrm{~cm}^{2}$ was used in the trailing edge experiments (see Figure 2). The separate flow nozzle system, described in detail in ref. 11, is approximately an $11 \%$ model of a typical commercial aircraft BPR 5 engine exhaust nozzle. Stereolithography shells [see Figure 3 (a)] were attached to the exterior of the core (base) nozzle to increase the nozzle trailing edge thickness. All transitional steps between the stereolithography shells and the base nozzle were smoothed with high temperature silicon sealant. The resulting average trailing edge thickness was determined from measurements made at 8 circumferential locations at the nozzle trailing edge. Five trailing edge thicknesses were investigated: $0.38 \mathrm{~mm}$ (base nozzle), $0.89 \mathrm{~mm}, 1.17 \mathrm{~mm}, 1.52$ $\mathrm{mm}$, and $3.17 \mathrm{~mm}$. A variable thickness (crenellated) shell with six, equally spaced "fingers" extending to the trailing edge was also tested [see Figure 3 (b)]. The trailing edge thickness of the fingers was $3.17 \mathrm{~mm}$ and the thickness between the fingers was equal to that of the base nozzle. The circumferential length of each finger was 1.9 $\mathrm{cm}$.

For the trailing edge experiments, the stagnation temperatures in the fan and core streams were maintained at $311^{\circ} \mathrm{K}$ and the NPR of each air stream was varied independently between 1.113 (jet velocity of $137 \mathrm{~m} / \mathrm{s}$ ) and 1.87 (jet velocity of $320 \mathrm{~m} / \mathrm{s}$ ). The tunnel Mach number was maintained at 0.1 . When standard takeoff conditions are used for this model system for a representative engine cycle, the fan velocity is $326 \mathrm{~m} / \mathrm{s}$ at $359^{\circ} \mathrm{K}$ and the core velocity is $450 \mathrm{~m} / \mathrm{s}$ at $828^{\circ} \mathrm{K}$ as discussed in ref. 11 .

Boundary layer measurements were taken at the outer trailing edge of the core base nozzle with a $0.15 \mathrm{~mm}$ stagnation boundary layer probe. For a core NPR equal to 1.488 and a fan NPR equal to 1.357 , the boundary layer thickness was equal to $2.5 \mathrm{~mm}$. The boundary layer measurements were made with both streams at $311^{\circ} \mathrm{K}$. 


\section{Results}

\section{A. Constant Thickness Trailing Edge}

\section{Thickness Effect}

Acoustic measurements were made for equal fan and core velocities between $137 \mathrm{~m} / \mathrm{s}$ and $320 \mathrm{~m} / \mathrm{s}$. Tones were not present for the base nozzle (trailing edge thickness equal to $0.38 \mathrm{~mm}$ ) at any operating condition investigated. For the remaining core nozzle trailing edges, tones were present for all equal velocity operating conditions.

The plots in Figure 4 were obtained for a trailing edge thickness equal to $0.89 \mathrm{~mm}$ and velocities greater than, or equal to, $198 \mathrm{~m} / \mathrm{s}$. Spectra corresponding to lower flow velocities have been omitted from the figure for clarity. All observation angles are measured relative to the upstream jet axis. Multiple tones with almost equal magnitude appear in the spectra for flow velocities below $229 \mathrm{~m} / \mathrm{s}$. When the flow velocity is greater than or equal to $229 \mathrm{~m} / \mathrm{s}$, only a single tone appears at each operating condition. At the highest speed tested $(320 \mathrm{~m} / \mathrm{s})$, the level of the tone begins to become equal to the broadband noise of the jet. The amplitudes of the tones relative to the broadband noise are highest for observation angles less than, or equal to, $90^{\circ}$. For the thickest trailing edge nozzles $(1.52 \mathrm{~mm}$ and $3.17 \mathrm{~mm}$ ), significant trailing edge tones are identified in the spectra measured at all angles. The frequencies of the tones increase with increasing flow velocity.

The spectra obtained for three of the trailing edge shells and the base nozzle at a single operating condition are shown in Fig. 5. As the trailing edge thickness increases, the amplitude of the tone increases and the frequency of the tone decreases. Plots of the frequency versus velocity and thickness are shown in Figures 6 and 7, respectively. The frequency scales linearly with flow velocity and almost linearly with thickness except for the thickest trailing edge $(3.17 \mathrm{~mm})$.

\section{Velocity Ratio Effect}

Previous investigations have indicated a connection between the ratio of the velocities across the nozzle lip and the production of tones ${ }^{4,5}$. However, the coannular nozzle data in these references are limited to velocity ratios, $\mathrm{V}_{\mathrm{r}}$, between 0 and 1 and core nozzle trailing edges that are quite thick (around $3.2 \mathrm{~mm}$ ). The velocity ratio is defined as

$V_{r}=\frac{V_{f}}{V_{c}}$, where $\mathrm{V}_{\mathrm{c}}$ is the core velocity and $\mathrm{V}_{\mathrm{f}}$ is the fan velocity. Larger velocity ratios occur for inverted velocity profiles and most scale model tests are typically performed with nozzles thinner than $3.2 \mathrm{~mm}$. In the present investigation, two sets of velocity ratio studies were performed for each installed core shell and for the base nozzle. In the first study, the core velocity was held constant at $320 \mathrm{~m} / \mathrm{s}$ and the velocity ratio was varied between 0.43 and 1.0 by altering the fan stream velocity. In the second set of studies, the fan stream was held constant at $320 \mathrm{~m} / \mathrm{s}$ and the velocity ratio was varied between 1.0 and 2.33 by altering the core stream velocity.

The power spectra for core nozzle trailing edges equal to $3.17 \mathrm{~mm}$ and $0.89 \mathrm{~mm}$ and for velocity ratios between 0.62 and 1 are shown in Figures 8 and 9. For the thickest trailing edge, the production of tones begins at a velocity ratio of 0.81 and the level of the tones is well above the local broadband noise for all observation angles. For the thinner trailing edge $(0.89 \mathrm{~mm})$, tones appear at a velocity ratio of 0.91 and radiate in the forward quadrant. As the trailing edge thickness increases, the magnitude of the tones increases. The frequencies of the tones appear to be relatively unaffected by changes in velocity ratio and increase with trailing edge thickness indicating that the core velocity and the trailing edge thickness are the important scaling parameters for this type of phenomenon. No tones were produced at any velocity ratio for the base nozzle.

Plots of the power spectra for core nozzle trailing edges equal to $3.17 \mathrm{~mm}$ and $0.89 \mathrm{~mm}$ and for velocity ratios between 1.1 and 2.33 are shown in Figures 10 and 11. Tones first appear at a velocity ratio equal to 1.61 and this appears to be independent of the thickness of the nozzle lip. As the velocity ratio is decreased, the magnitude of the tones first increases then begins to decrease relative to the background noise at a velocity ratio of 1.10 . The frequencies change with velocity ratio (changing core velocity) and nozzle thickness. For a constant velocity ratio, the magnitude of the tones increases with increasing nozzle trailing edge thickness. No tones were present for the base nozzle.

\section{On-set of Tones and Scaling Considerations}

The condition corresponding to the on-set of tones is defined here as the operating condition and nozzle trailing edge thickness that result in tones exceeding the local broadband noise by $5 \mathrm{~dB}$. The plot in Figure 12 shows the regions where tones are produced for a range of velocity ratios and nozzle lip thicknesses. From the plot, it is evident that, once the nozzle lip thickness is great enough that tones are produced, the operating conditions leading 
to the production of tones is relatively insensitive to nozzle lip thickness although tones are first produced at a slightly lower velocity ratio for the thickest trailing edge than for the other trailing edges tested.

The data were scaled by the Strouhal number, $S t=\frac{f t}{V_{c}}$, due to the dependency of the discrete frequency tone, $\mathrm{f}$, on the core velocity. The length scale, $t$, is given by the nozzle trailing edge thickness. When a relatively broad peak occurred in the spectrum, the center frequency of the peak was chosen for the Strouhal calculations. The data were not well represented by a Strouhal number based on the average velocity of the two streams.

A plot of the Strouhal number versus the nozzle trailing edge thickness for a range of velocity ratios is shown in Figure 13. The data show that the Strouhal number is a function of both velocity ratio and nozzle trailing edge thickness. For velocity ratios greater than one and constant values of $t$, the Strouhal number increases with increasing velocity ratio. For all values of the velocity ratio, the Strouhal number increases with increasing nozzle trailing edge thickness. Olsen and $\mathrm{Karchmer}^{5}$ found a Strouhal number equal to 0.20 in their trailing edge studies using a coannular nozzle.

The average Strouhal numbers were calculated from all measurements made for each trailing edge thickness in the velocity ratio range of 0.81 to 1.41 . The maximum deviation of any Strouhal number from the mean value was within $10 \%$ of the mean for all $\mathrm{V}_{\mathrm{r}}$. Larger deviations from the mean value occurred for a velocity ratio of 1.61 . By extrapolating the data in Figure 13, it is expected that, if the base nozzle produced tones, the resulting Strouhal number would be equal to approximately 0.15 leading to frequencies between $70,000 \mathrm{~Hz}$ and $125,000 \mathrm{~Hz}$ for the velocities tested.

\section{Broadband Increases}

The effect of trailing edge thickness on high frequency noise for a single operating condition $\left(\mathrm{V}_{\mathrm{r}}=1.24\right)$ is shown in Figure 14. For this condition, tones are produced for a nozzle lip thickness greater than, or equal to, 0.89 $\mathrm{mm}$. Increases in noise above that of the base nozzle are observed at frequencies greater than that of the discrete tone. The magnitude of broadband noise increases with increasing nozzle lip thickness. The impact of the trailing edge is more pronounced in the forward quadrant than in the aft quadrant although the thickest trailing edge results in significant increases in high frequency broadband noise at all observation angles.

A plot of the spectra obtained for a velocity ratio of 0.72 is shown in Figure 15. For this operating condition, tones are not produced for any nozzle trailing edge thickness. Slight increases in high frequency noise with increasing nozzle thickness are observed in the forward quadrant. For an observation angle of $61^{\circ}$ and a frequency of $45,000 \mathrm{~Hz}$, there is roughly a $0.7 \mathrm{~dB}$ increase between the $0.38 \mathrm{~mm}$ trailing edge (base nozzle) and the $0.89 \mathrm{~mm}$ trailing edge and a $2.2 \mathrm{~dB}$ increase between the $0.38 \mathrm{~mm}$ trailing edge and the $3.17 \mathrm{~mm}$ trailing edge. There is no noticeable effect of trailing edge thickness on broadband noise at aft angles when no tones are produced.

The narrowband data for a velocity ratio of 0.72 was scaled to full scale using a scale model factor of 9 and a sideline distance of $1783 \mathrm{ft}$. The extrapolated one-third octave band data are shown in Figure 16. Increases in broadband noise with increasing nozzle lip thickness are noted for frequencies above $1000 \mathrm{~Hz}$ in the forward quadrant. For the $3160 \mathrm{~Hz}$ band, the sound pressure level difference between the base nozzle $(0.38 \mathrm{~mm}$ trailing edge) and the $0.89 \mathrm{~mm}$ trailing edge is $1.2 \mathrm{~dB}$, and the difference between the base nozzle and the $3.17 \mathrm{~mm}$ trailing edge is $3.4 \mathrm{~dB}$ for an observation angle of $61^{\circ}$. Increases in high frequency broadband noise are quite small at aft angles. The EPNL is also affected by trailing edge thickness with an increase of $0.6 \mathrm{EPNdB}$ for the $0.89 \mathrm{~mm}$ trailing edge nozzle relative to the base nozzle, and an increase of $0.9 \mathrm{EPNdB}$ for the $3.17 \mathrm{~mm}$ trailing edge nozzle relative to the base nozzle. Since small changes in EPNL are often important when evaluating noise reduction devices, the effect of trailing edge thickness can be significant for full scale predictions made from scale model tests on noise reduction devices.

\section{B. Crenellated Trailing Edge}

One concept for reducing the effect of trailing edge thickness is to crenellate the nozzle as shown in Figure 3 (b). If the resulting increase in noise is within an acceptable limit, the thicker regions of the nozzle can be used to install mixing devices. Acoustic measurements were taken for the crenellated nozzle described in Section II, for velocity ratios between 0.62 and 1.61. Discrete tones were not produced for any of the conditions tested possibly due to a reduction in the correlation length of the distributed sources at the nozzle trailing edge. The impact of the crenellated design on the broadband noise is discussed in this section.

The spectra in Figure 17 were obtained for a velocity ratio of 0.72 . Increases in high frequency noise for the crenellated nozzle are not as significant as that for the $3.17 \mathrm{~mm}$ constant thickness trailing edge. The crenellated 
nozzle results in an increase of roughly $0.5 \mathrm{~dB}$ over that of the base nozzle at a frequency of $45,000 \mathrm{~Hz}$ and an observation angle of $61^{\circ}$. No high frequency increase is observed at $90^{\circ}$ and at aft angles.

Plots of the extrapolated full-scale one-third octave data using the scaling factors described previously are shown in Figure 18. An increase in broadband noise occurs for the crenellated nozzle over that of the base nozzle. The broadband increase for the $3160 \mathrm{~Hz}$ band of the crenellated nozzle relative to that of the base nozzle is $1.3 \mathrm{~dB}$ at an observation angle of $61^{\circ}$, a significant improvement over that of the $3.17 \mathrm{~mm}$ constant thickness trailing edge. The increase in EPNL for the crenellated nozzle relative to the base nozzle is $0.5 \mathrm{EPNdB}$.

\section{Conclusions}

The effect of core nozzle trailing edge thickness is measured in a coannular nozzle system for velocity ratios between 0.43 and 2.33. No tones are produced for a trailing edge thickness equal to $0.38 \mathrm{~mm}$. For a trailing edge thickness greater than or equal to $0.89 \mathrm{~mm}$, tones are produced for velocity ratios between 0.9 and 1.61 . For a constant trailing edge thickness, the frequency of the tones increases with core velocity. The Strouhal number based on the core velocity increases with increasing nozzle trailing edge thickness. Average values for the Strouhal number calculated for each core nozzle trailing edge thickness are between 0.16 and 0.2 . The radiation of discrete tones is most prominent in the forward quadrant except for the thickest trailing edge where trailing edge tones are often observed at all observation angles.

Increases in high frequency broadband acoustic radiation with increasing trailing edge thickness are observed for operating conditions where tones are produced and for conditions where tones are not produced. For non-tone producing conditions, extrapolated sideline acoustic radiation for a full scale model showed an increase of $1.2 \mathrm{~dB}$ for the $0.89 \mathrm{~mm}$ trailing edge nozzle and $3.4 \mathrm{~dB}$ for the $3.17 \mathrm{~mm}$ trailing edge nozzle relative to the $0.38 \mathrm{~mm}$ base nozzle for the $3160 \mathrm{~Hz}$ band and an observation angle of $61^{\circ}$. Increases in EPNL with increasing trailing edge thickness are also observed.

No tones are produced for the variable thickness (crenellated) trailing edge nozzle. High frequency broadband noise is also reduced from that of the corresponding constant thickness nozzle.

\section{Acknowledgements}

The authors thank the Jet Noise Laboratory staff for their contributions to the planning, facility operation, and data acquisition required in this experimental effort.

\section{References}

${ }^{1}$ Powell, A., "On the Aerodynamic Noise of a Rigid Flat Plate Moving at Zero Incidence," J. Acoust. Soc. Am., Vol. 31 , No. 12,1959 , pp. $1649-1653$.

${ }^{2}$ Ffowcs Williams, J. E., and Hall, L. H., "Aerodynamic Sound Generation by Turbulent Flow in the Vicinity of a Scattering Half Plane,” J. Fluid Mech., Vol. 40, part 4, 1970, pp. 657 - 670.

${ }^{3}$ Brooks, T. F., Pope, D. S., and Marcolini, A., “Airfoil Self-Noise and Prediction,” NASA Ref. Pub. 1218, 1989.

${ }^{4}$ Olsen, W. A., Guitierrez, O., and Dorsch, R. G., "The Effect of Nozzle Inlet Shape, Lip Thickness, and Exit Shape and Size on Subsonic Jet Noise," AIAA Paper No. 73-187, 1973.

${ }^{5}$ Olsen, W., and Karchmer, A., "Lip Noise Generated by Flow Separation from Nozzle Surfaces," NASA TM X-71859, 1976.

${ }^{6}$ Ffowcs Williams, J. E., and Gordon, C. G., "Noise of Highly Turbulent Jets at Low Exhaust Speeds,” AIAA J., Vol. 3, No. 4, pp. $791-793,1965$.

${ }^{7}$ Leppington, F. G., "Scattering of Quadrupole Sources Near the End of a Rigid Semi-Infinite Circular Pipe,” ARC C. P. No. 1195, 1972.

${ }^{8}$ Brinich, P. F., Boldman, D. R., and Goldstein, M. E., "Vortex Shedding from a Blunt Trailing Edge with Equal and Unequal External Mean velocities," NASA TN D-8034, 1975.

${ }^{9}$ Ahuja, K. K., and Blakney, D. F., “Tone Excited Jets, Part IV: Acoustic Measurements,” J. Sound and Vib., Vol 102 (1), 93 $-117,1985$.

${ }^{10}$ Amiet, R. K., "Refraction of Sound by a Shear Layer," AIAA Paper No. 77-54, (1977).

${ }^{11}$ Thomas, R., and Kinzie, K., "Jet-pylon Interaction of High Bypass Ratio Separate Flow Nozzle Configurations," AIAA Paper No. 2004-2827, (2004). 

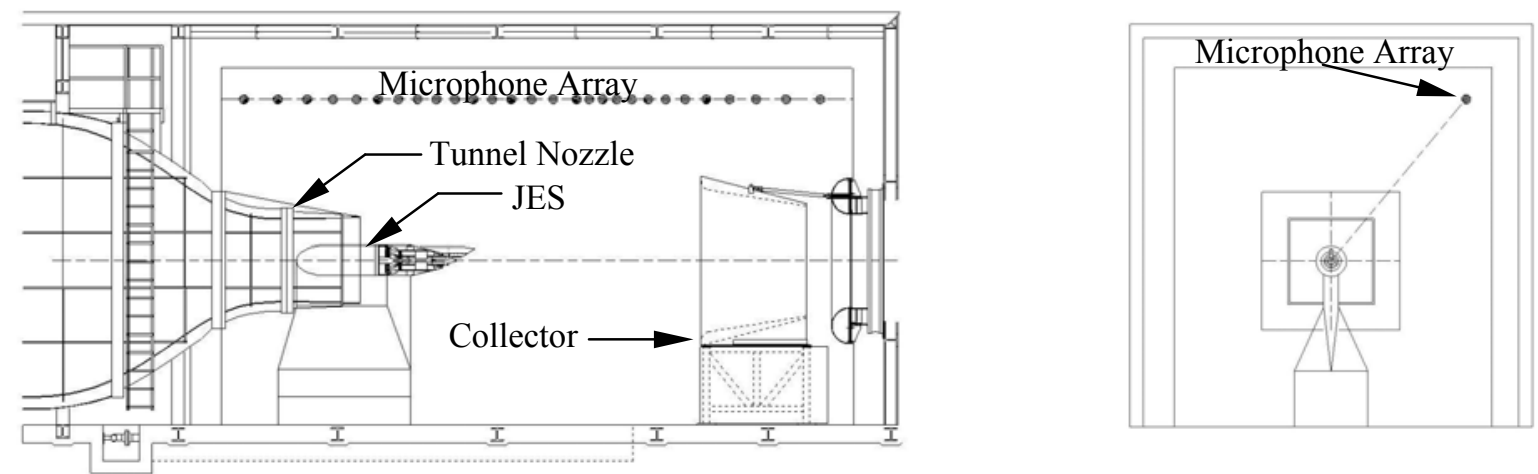

Figure 1. A schematic of the Low Speed Aeroacoustics Wind Tunnel (LSAWT) and the Jet Engine Simulator (JES).

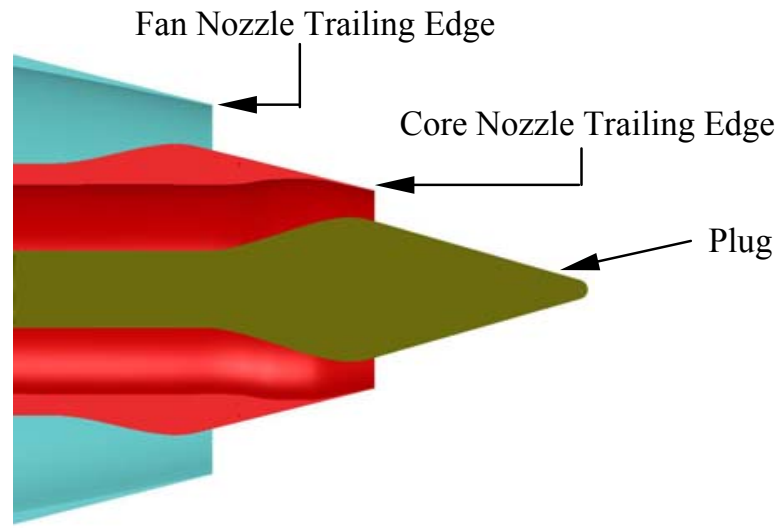

Figure 2. A schematic of the base nozzle used in the nozzle trailing edge experiments.

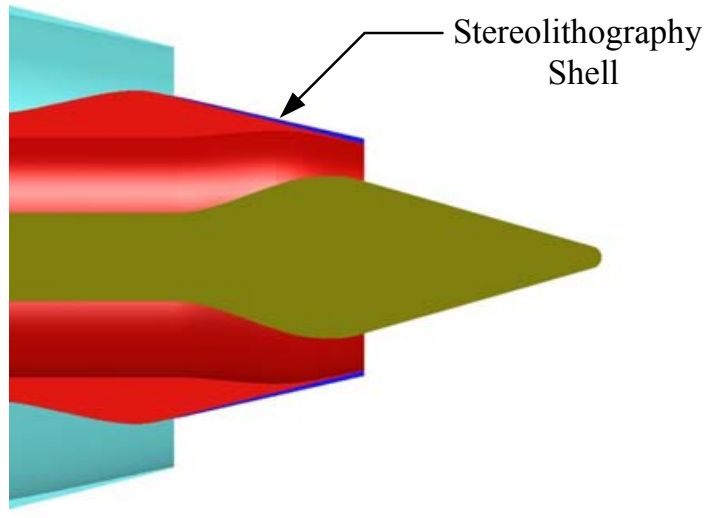

(a)

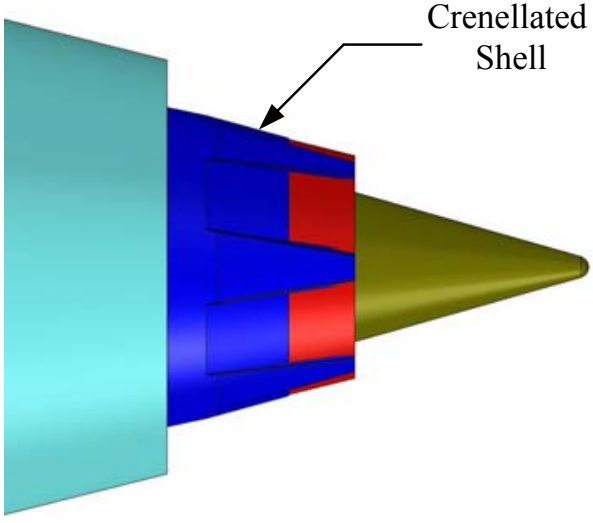

(b)

Figure 3. The stereolithography shells used for (a) the constant thickness trailing edge studies and (b) the crenellated trailing edge studies. 


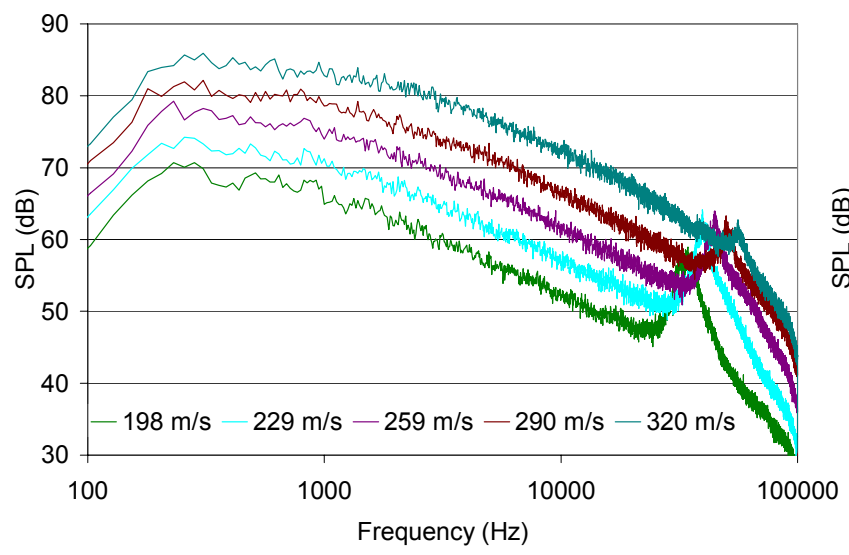

(a)

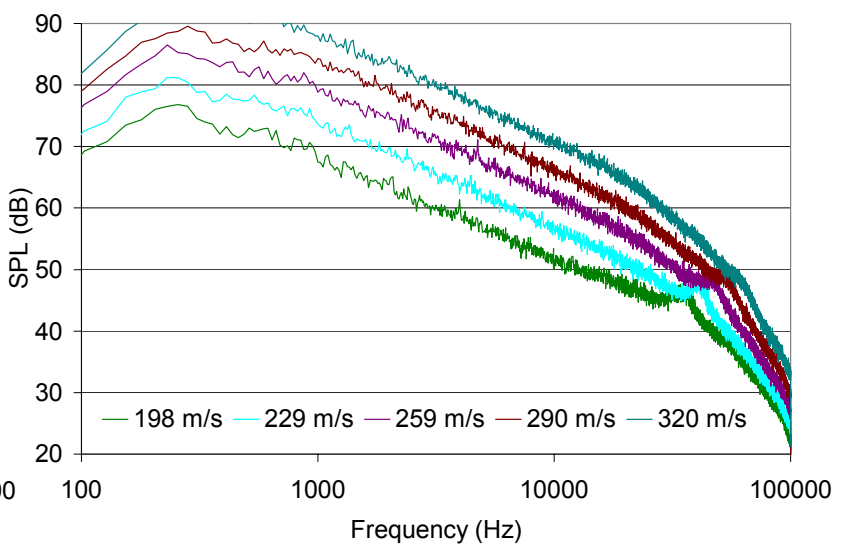

(b)

Figure 4. The power spectra obtained for a nozzle trailing edge thickness of $0.89 \mathrm{~mm}$ and equal core and fan velocities at observation angles of (a) $61^{\circ}$ and (b) $141^{\circ}$.

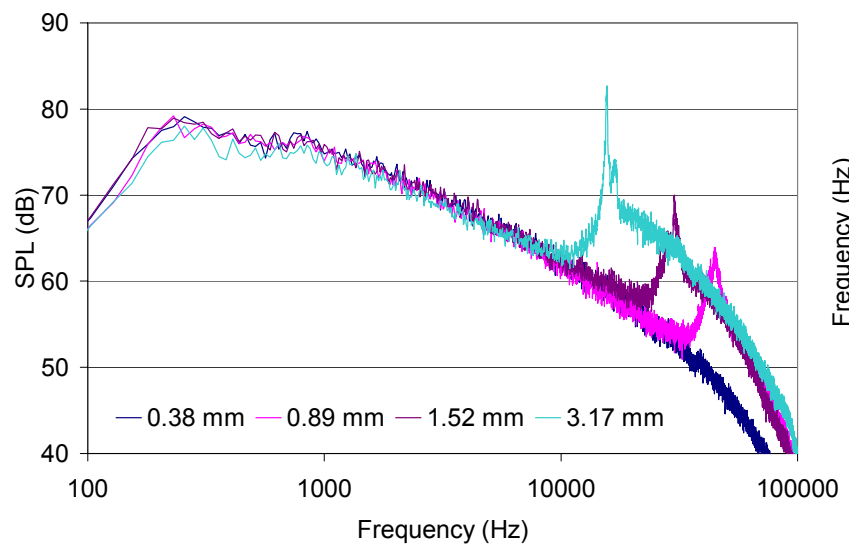

Figure 5. The power spectrum obtained for fan and core velocities equal to $260 \mathrm{~m} / \mathrm{s}$ and an observation angle of $61^{\circ}$.

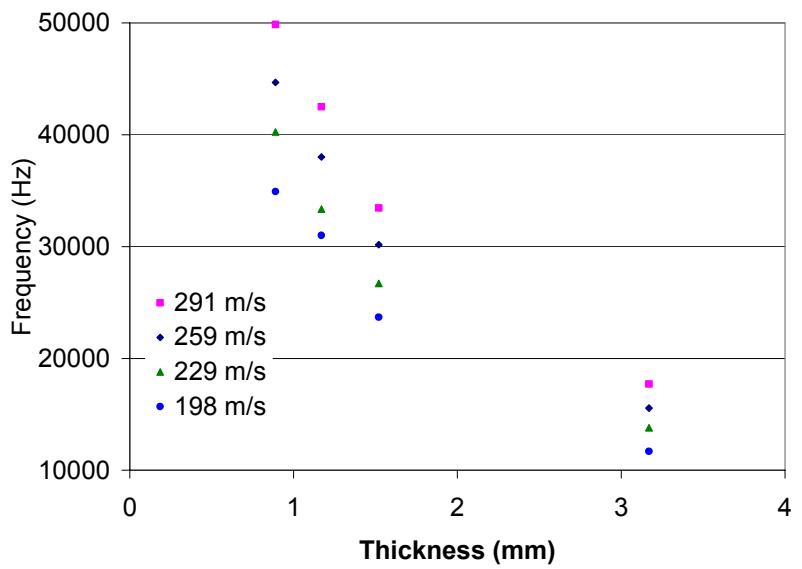

Figure 7. The frequency of the discrete tone as a function of thickness for velocities between $198 \mathrm{~m} / \mathrm{s}$ and $291 \mathrm{~m} / \mathrm{s}$.

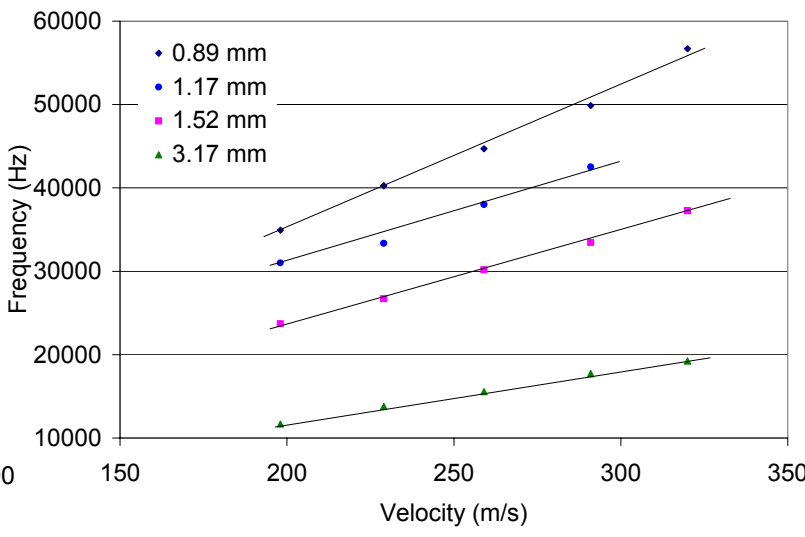

Figure 6. The frequency of the discrete tone as a function of flow velocity for a trailing edge thickness between $0.89 \mathrm{~mm}$ and $3.17 \mathrm{~mm}$. 


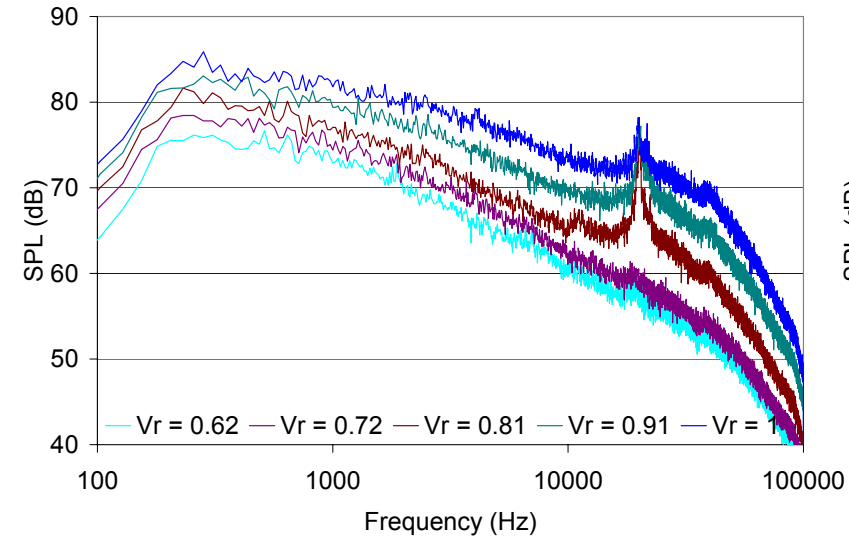

(a)

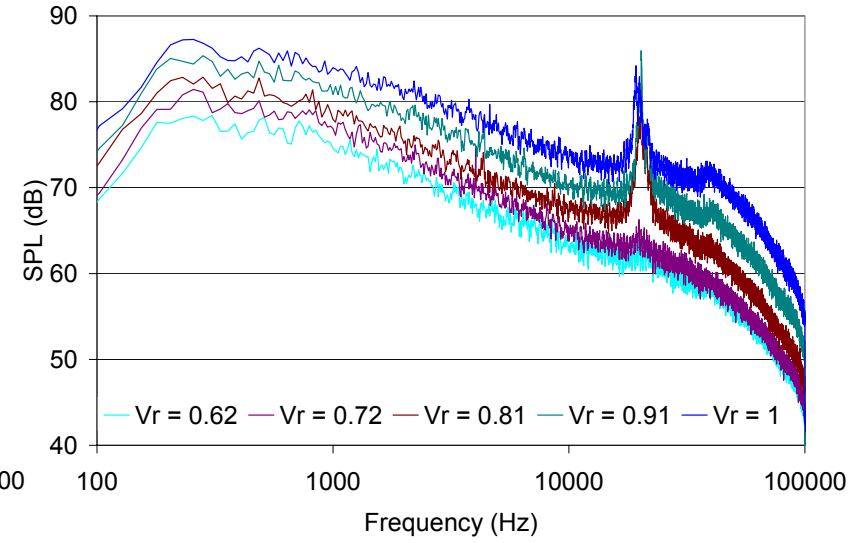

(b)

Figure 8. The power spectra obtained for a core velocity of $320 \mathrm{~m} / \mathrm{s}$, a trailing edge thickness of $3.17 \mathrm{~mm}$, and (a) a $61^{\circ}$ observation angle and (b) a $90^{\circ}$ observation angle

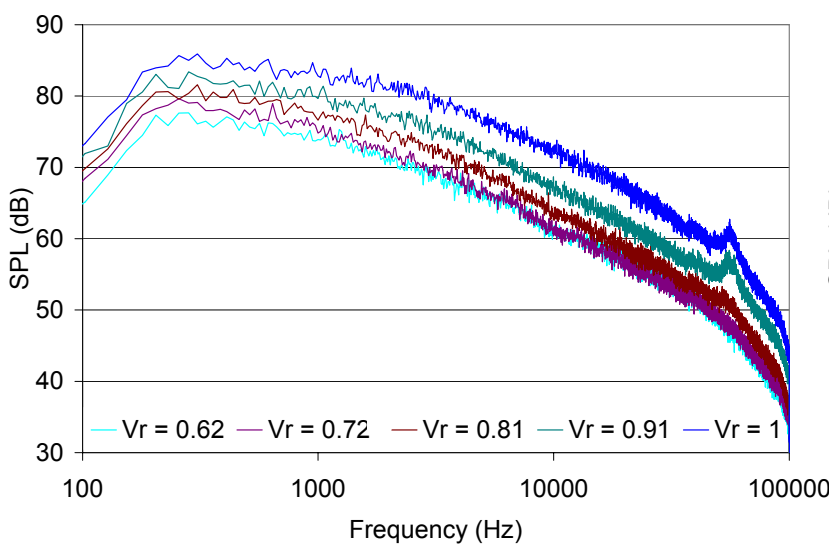

(a)

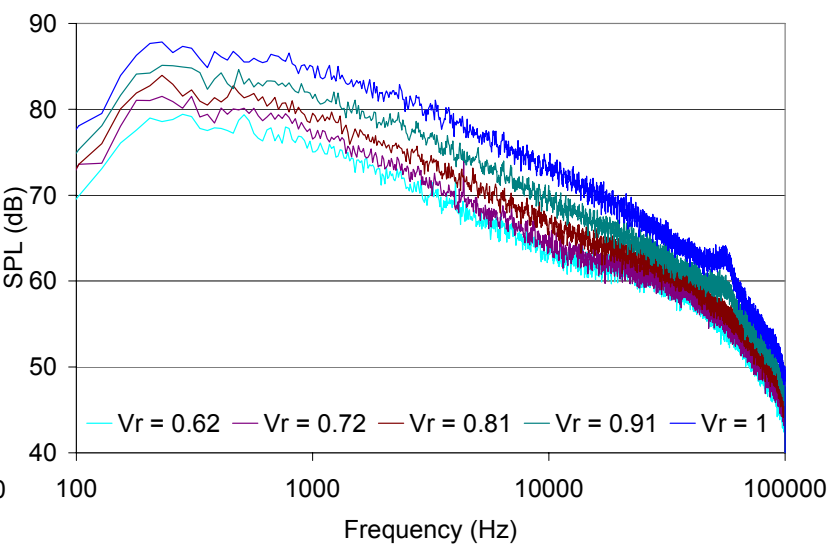

(b)

Figure 9. The power spectra obtained for a core velocity of $320 \mathrm{~m} / \mathrm{s}$, a trailing edge thickness of $0.89 \mathrm{~mm}$, and (a) a $61^{\circ}$ observation angle and (b) a $90^{\circ}$ observation angle.

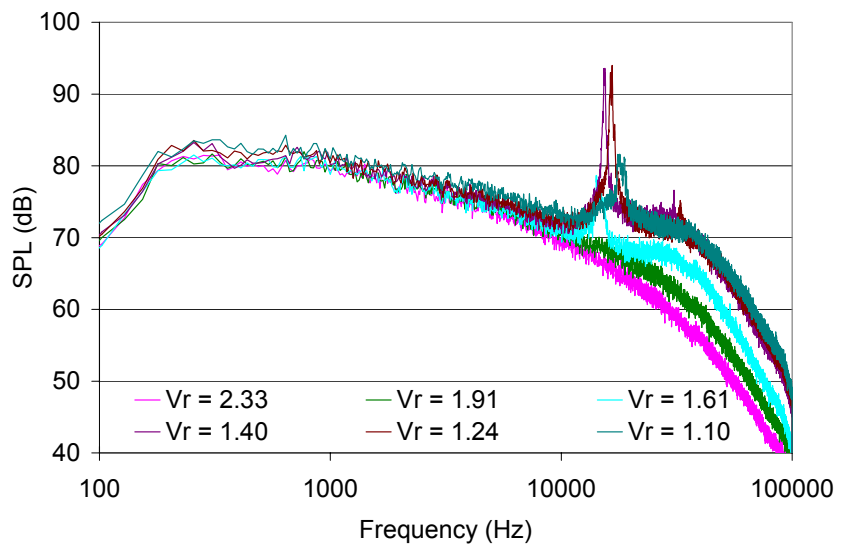

(a)

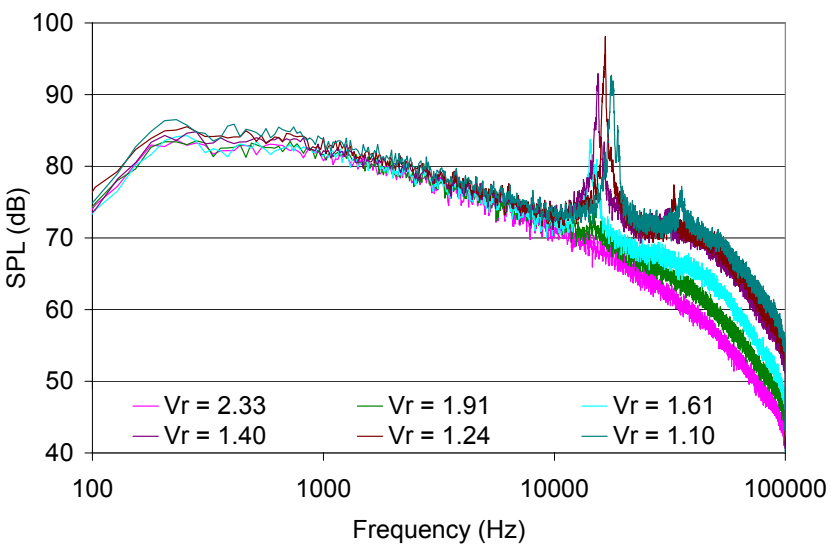

(b)

Figure 10. The power spectra obtained for a fan velocity of $320 \mathrm{~m} / \mathrm{s}$, a trailing edge thickness of $3.17 \mathrm{~mm}$, and (a) a $61^{\circ}$ observation angle and (b) a $90^{\circ}$ observation angle. 


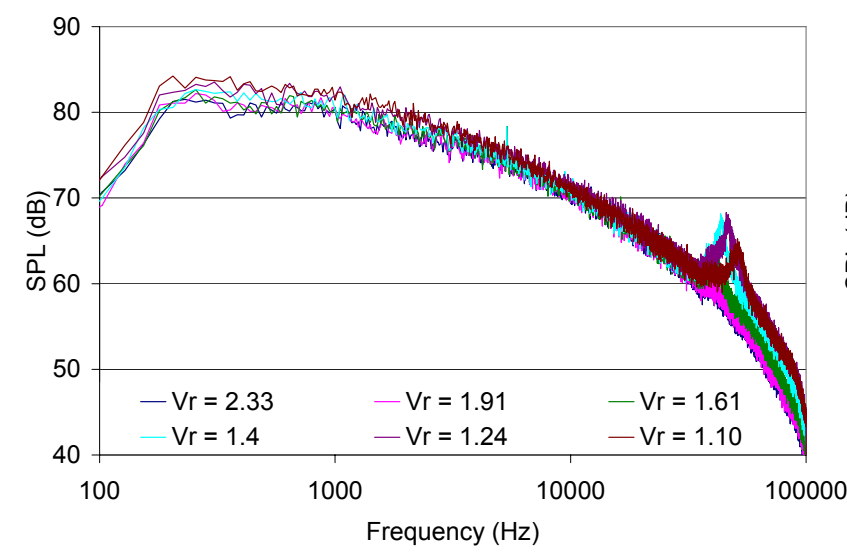

(a)

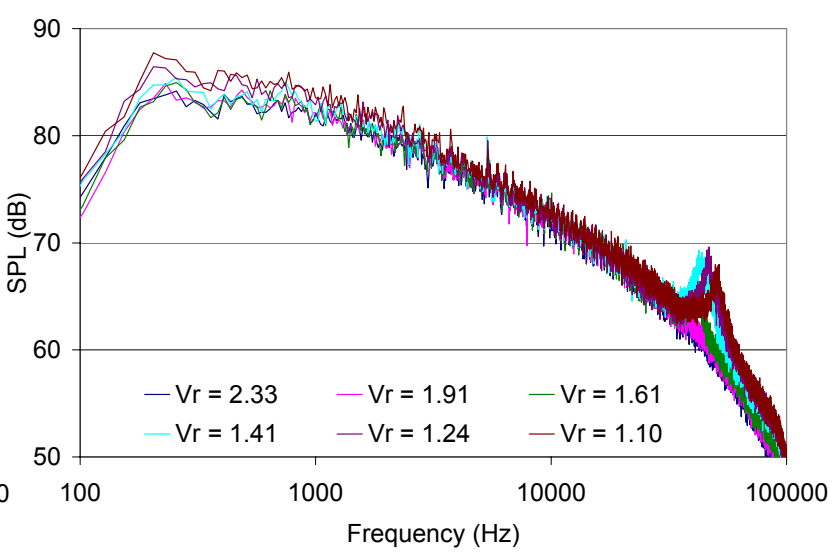

(b)

Figure 11. The power spectra obtained for a fan velocity of $320 \mathrm{~m} / \mathrm{s}$, a trailing edge thickness of $0.89 \mathrm{~mm}$, and (a) a $61^{\circ}$ observation angle and (b) a $90^{\circ}$ observation angle.

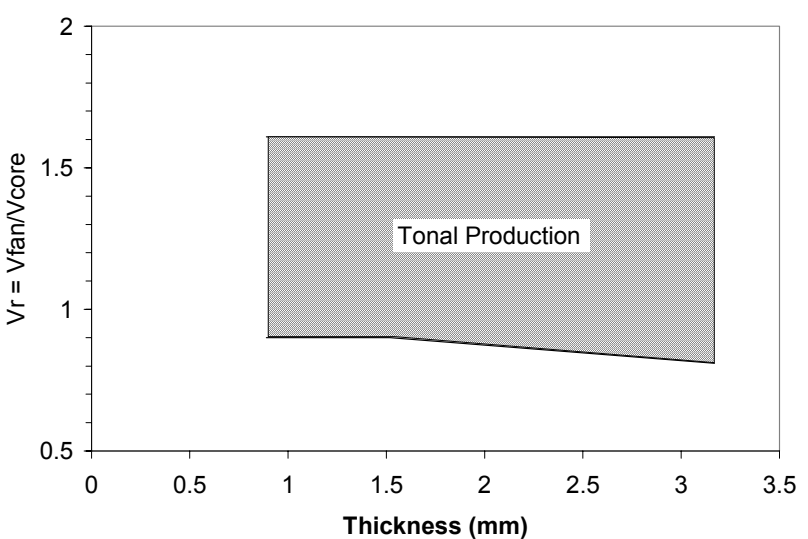

Figure 12. The region where tones are produced as a function of velocity ratio and trailing edge thickness.

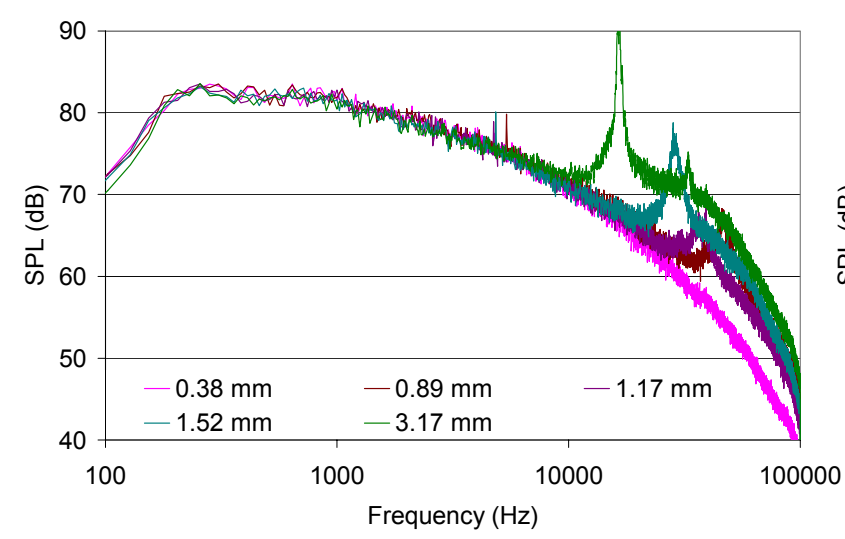

(a)

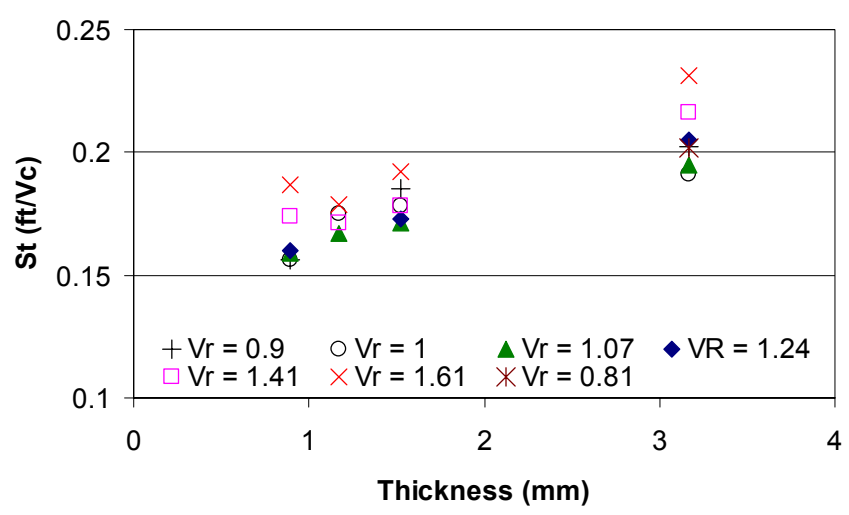

Figure 13. The change in Strouhal number based on core velocity as a function of trailing edge thickness and velocity ratio.

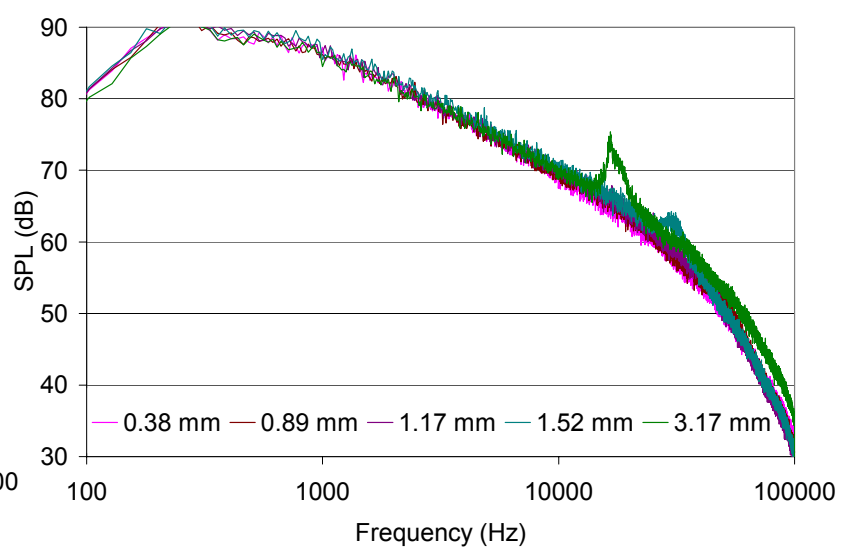

(b)

Figure 14. The power spectra obtained for a fan velocity of $320 \mathrm{~m} / \mathrm{s}$ and a core velocity of $259 \mathrm{~m} / \mathrm{s}$ for a range of trailing edges and (a) a $61^{\circ}$ observation angle and (b) a $141^{\circ}$ observation angle. 


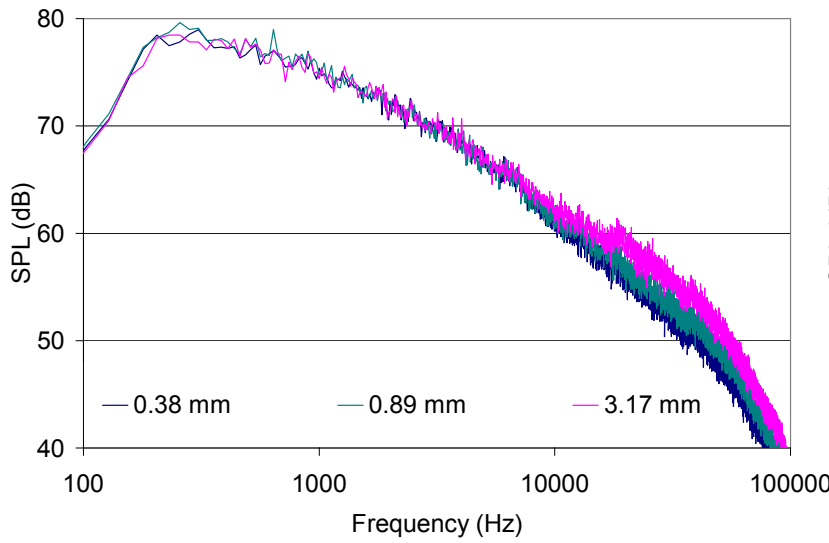

(a)

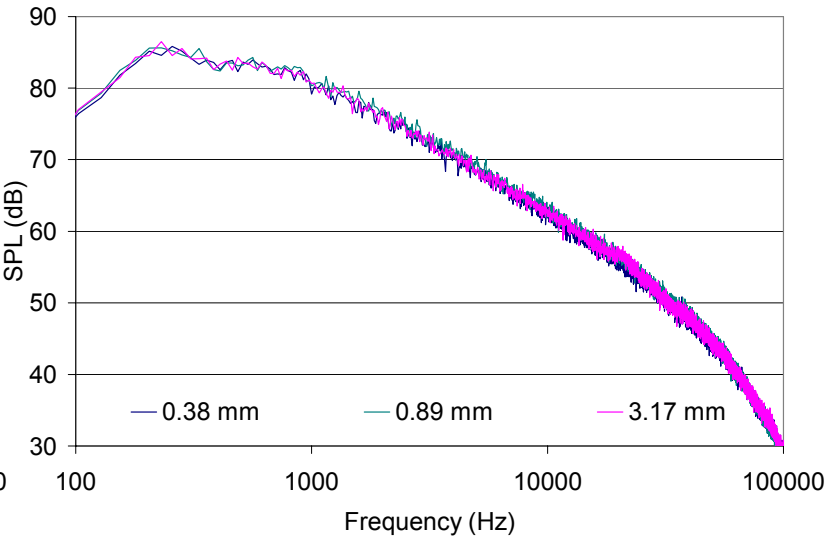

(b)

Figure 15. The power spectra obtained for a fan velocity of $229 \mathrm{~m} / \mathrm{s}$ and a core velocity of $320 \mathrm{~m} / \mathrm{s}$ for a range of nozzle trailing edges and (a) a $61^{\circ}$ observation angle and (b) a $141^{\circ}$ observation angle.

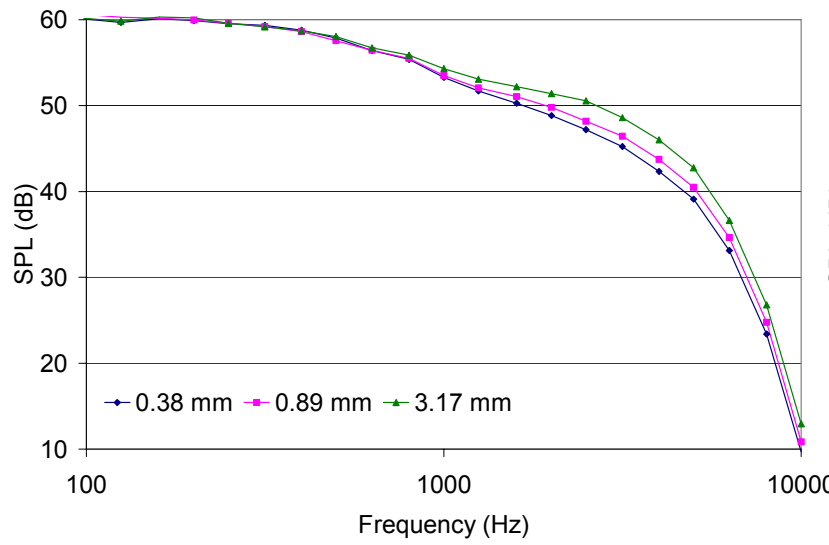

(a)

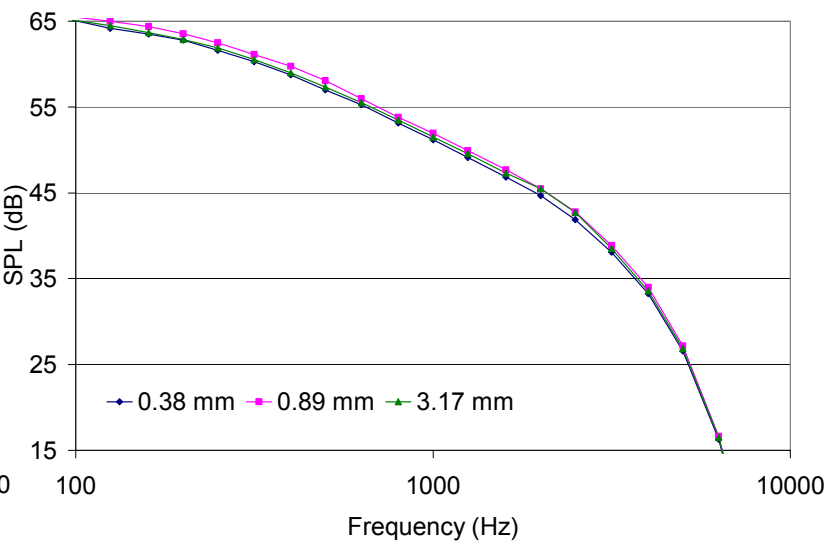

(b)

Figure 16. Extrapolated full scale power spectral data obtained for a fan velocity of $229 \mathrm{~m} / \mathrm{s}$ and a core velocity of $320 \mathrm{~m} / \mathrm{s}$ for a range of trailing edges and (a) a $61^{\circ}$ observation angle and (b) a $141^{\circ}$ observation angle.

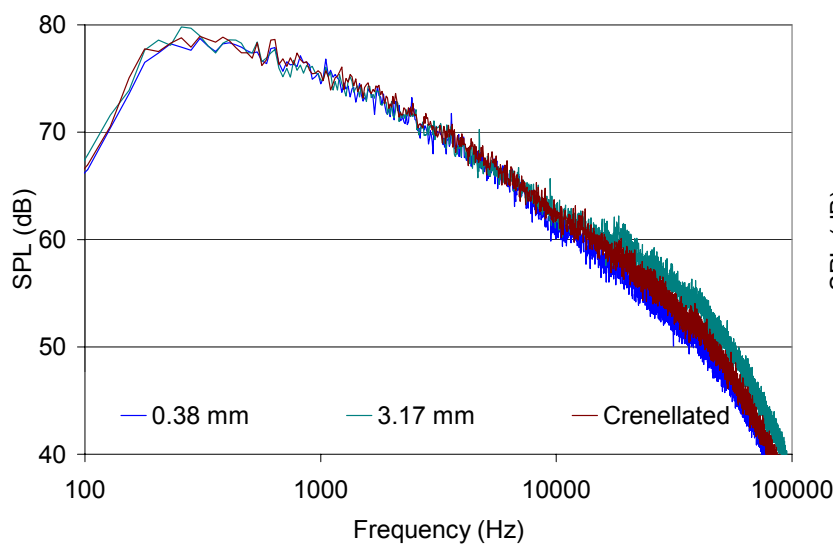

(a)

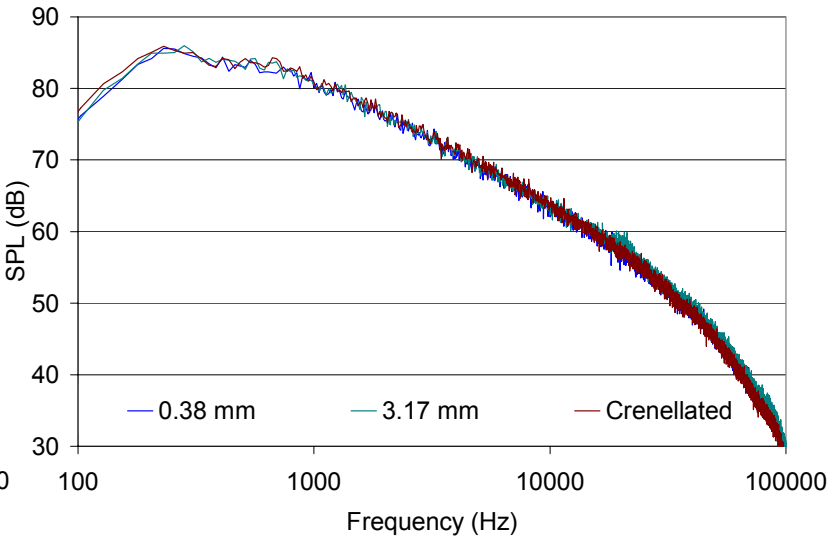

(b)

Figure 17. The power spectra obtained for a fan velocity of $229 \mathrm{~m} / \mathrm{s}$ and a core velocity of $320 \mathrm{~m} / \mathrm{s}$ for constant thickness and crenellated trailing edges and for (a) a $61^{\circ}$ observation angle and (b) a $138^{\circ}$ observation angle. 


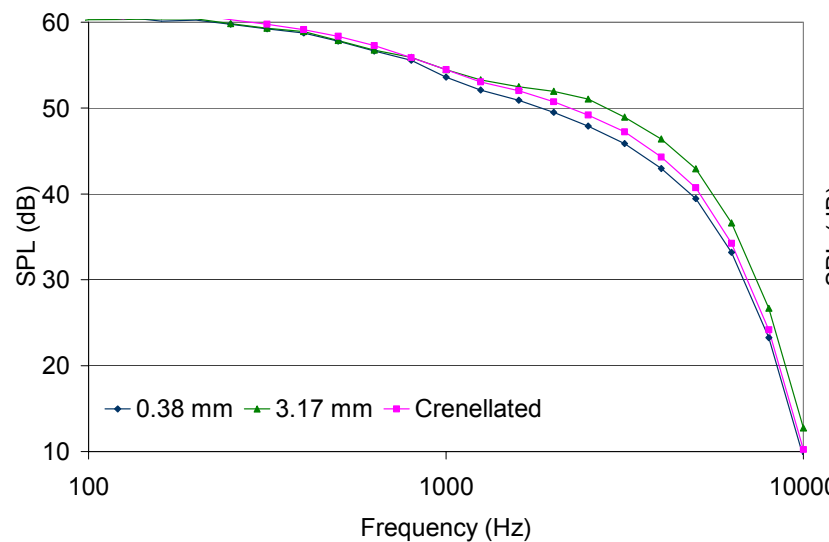

(a)

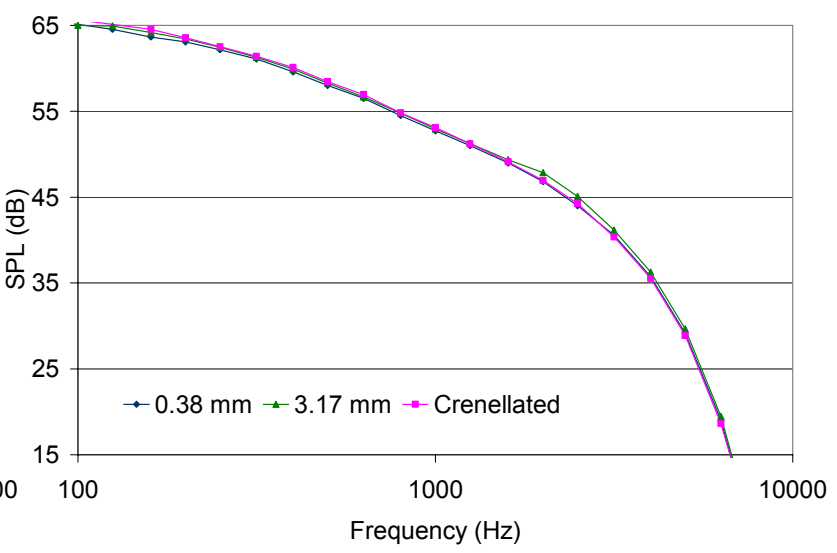

(b)

Figure 18. Extrapolated full scale power spectral data obtained for a fan velocity of $229 \mathrm{~m} / \mathrm{s}$ and a core velocity of $320 \mathrm{~m} / \mathrm{s}$ for constant thickness and crenellated trailing edges and for (a) a $61^{\circ}$ observation angle and (b) a $138^{\circ}$ observation angle. 